\title{
Haploidentical Transplantation in Children with Acute Leukemia: The Unresolved Issues
}

\author{
Sarita Rani Jaiswal ${ }^{1,2}$ and Suparno Chakrabarti ${ }^{1,2}$ \\ ${ }^{1}$ Department of Blood and Marrow Transplantation, Dharamshila Hospital and Research Centre, Vasundhara Enclave, \\ New Delhi 110096, India \\ ${ }^{2}$ Manashi Chakrabarti Foundation, Kolkata, India \\ Correspondence should be addressed to Sarita Rani Jaiswal; drsaritaranij@gmail.com
}

Received 24 December 2015; Accepted 21 February 2016

Academic Editor: Nelson J. Chao

Copyright (C) 2016 S. R. Jaiswal and S. Chakrabarti. This is an open access article distributed under the Creative Commons Attribution License, which permits unrestricted use, distribution, and reproduction in any medium, provided the original work is properly cited.

\begin{abstract}
Allogeneic hematopoietic stem cell transplantation (HSCT) remains a curative option for children with high risk and advanced acute leukemia. Yet availability of matched family donor limits its use and although matched unrelated donor or mismatched umbilical cord blood (UCB) are viable options, they fail to meet the global need. Haploidentical family donor is almost universally available and is emerging as the alternate donor of choice in adult patients. However, the same is not true in the case of children. The studies of haploidentical HSCT in children are largely limited to T cell depleted grafts with not so encouraging results in advanced leukemia. At the same time, emerging data from UCBT are challenging the existing paradigm of less stringent HLA match requirements as perceived in the past. The use of posttransplantation cyclophosphamide (PTCY) has yielded encouraging results in adults, but data in children is sorely lacking. Our experience of using PTCY based haploidentical HSCT in children shows inadequacy of this approach in younger children compared to excellent outcome in older children. In this context, we discuss the current status of haploidentical HSCT in children with acute leukemia in a global perspective and dwell on its future prospects.
\end{abstract}

\section{Introduction}

Despite marked improvement in the outcome of children with acute leukemia with first-line chemotherapy, a significant proportion of patients require allogeneic hematopoietic stem cell transplantation (HSCT) either in first remission (CR1) or beyond. In the BFM 95, about $12 \%$ of children diagnosed with acute lymphoblastic leukemia (ALL) went on to receive an allogeneic HSCT and the number increased in subsequent studies with introduction of MRD based risk stratification [1]. Likewise in the trials involving children with acute myeloid leukemia (AML), up to $30 \%$ of patients underwent an allogeneic transplantation [2]. In addition, allogeneic HSCT is the preferred modality of intervention beyond CR1. Thus, a conservative estimation would be that $25 \%$ of children with ALL and $40 \%$ of those with AML might require an allogeneic HSCT either in CR1 or beyond.

HLA matched family donor (MFD) remains the donor of choice in any indication for allogeneic HSCT. But with restricted family sizes, the chances of obtaining a MFD for a child are substantially reduced. Thus, alternate donor HSCT would be needed for the majority when an allogeneic HSCT is indicated and the focus of the transplant community in the past two decades has been on development of alternate donor sources.

\section{The Dilemma of HLA Matching: Time for Cord Blood As Well}

Developments in unrelated donor registries for both marrow and cord blood repositories have enabled progress in the field of allogeneic HSCT. Initial registry based studies had established equivalence between a mismatched unrelated cord blood transplantation (UCBT) and matched unrelated donor (MUD) transplantation [3]. HLA matching based on high resolution typing has improved the outcome of MUD transplants over the last two decades [4]. The limitations of 
North American and European registries in providing 8/8 HLA matched donors beyond the White Europeans have been largely addressed by the availability of $\geq 4 / 6$ HLA matched UCB units from the existing public cord blood banks [5]. Whilst low resolution typing for HLA-A and HLA$B$ and high resolution typing for DRB1 were deemed optimal for UCBT aiming for 4-6/6 HLA matched units, recent studies have challenged this notion [6-9]. A retrospective analysis on 803 patients, mostly children, showed the importance of HLA-C matching to reduce transplant related mortality (TRM), which was hitherto considered redundant [6]. At the same time, high resolution allele level matching for both single and double cord units was shown to reduce TRM [7, 9]. The impact of allele level or extended HLA-C matching was shown to be independent of the cell dose. These findings, if taken to cognizance, would restrict the availability of suitably matched UCB such as $\leq 2$ allele level mismatches including HLA-C. Thus, the attempts at optimizing the outcome of UCBT have pushed the quest for the third alternative that is HLA-haploidentical family donor (HFD) to the fore $[7,8]$.

\section{Haploidentical Family Donor: Always Present but Barely Noticed until Now}

The success of HSCT depends on establishment of bidirectional tolerance and compatibility of major HLA antigens is a prerequisite for the same. It has been aptly documented in the setting of unrelated donor HSCT that with each additional mismatch in HLA-A, HLA-B, HLA-C, or DRB1 the survival decreases by $10-20 \%$ [10-12]. Recent studies have highlighted the same regarding UCBT [8]. Early attempts at introducing haploidentical family donor as an alternate donor had failed miserably. Not unexpectedly, severe alloreactivity or graft rejection dominated the outcome and the concept of allograft from a HFD was not thought to be feasible [13].

\section{Megadoses of Purified CD34+ Cell Infusion: The Door Opened but Questions Remained}

The breakthrough came from murine experiments demonstrating the ability of megadoses of CD34+ cells to engraft across major HLA barriers [14-16]. This was translated to clinical reality by the group from Perugia when they reported 95\% engraftment with virtually no serious graft-versus-host disease (GVHD) without employment of GVHD prophylaxis, in patients with advanced leukemia [17]. This was possible due to advent of growth factor mobilized peripheral blood stem cell (PBSC) collection which enabled collection of large amounts of CD34 cells which was not hitherto possible from marrow grafts. The other advancement of technology provided the ability to purify CD34 cells via immunomagnetic techniques drastically reducing the $\mathrm{T}$ and $\mathrm{B}$ cell content of the graft. This approach was based on infusing CD34 cells in excess of $10 \times 10^{6} / \mathrm{kg}$ with a CD3 cell inoculum of $<1 \times 10^{5} / \mathrm{kg}$. In a pilot study on haploidentical HSCT with CD34 selected PBSC graft following myeloablative and immunoablative conditioning, Aversa et al. documented sustained engraftment in 41/43 patients with advanced leukemia without acute or chronic GVHD and $28 \%$ long term disease-free survival (DFS) [17]. Importantly, no pharmacological GVHD prophylaxis was employed. The study population included both adults and children with an age range of 4 to 53 years. However, the major drawback of this approach was delayed immune reconstitution resulting in mortality from opportunistic infections in about $40 \%$ of the patients. The reconstitution of CD4 T cells was delayed beyond 12 months in the surviving patients. In a study on 39 children employing a similar approach, 36 patients engrafted promptly with little or no GVHD [18]. The DFS was $28 \%$ and TRM was $34 \%$. Interestingly, immune reconstitution (IR) was noted to be better in those receiving $>20 \times 10^{9} / \mathrm{kg}$ CD34 cells. Subsequent studies by the Perugia group showed further improvement in outcome over the next decade, but TRM remained a major concern which was attributable to delayed IR [19-21]. Two studies from the UK highlighted similar findings with better results in patients in CR than those who were not in remission $[22,23]$. The outcome with this approach was remarkably better in patients with AML as compared to those with ALL [19].

An EBMT Pediatric Disease Working Party survey on 127 children with ALL transplanted between 1995 and 2004 revealed some interesting facts [24]. They found that transplants carried out by centres performing more than 231 allografts in the specified period with a median of $8 \mathrm{HFD}$ yielded a DFS of $39 \%$ compared to only $15 \%$ in those performing less than 231 allografts with a median of one HFD transplant. There was a trend towards lower relapse incidence (RI) and DFS amongst those receiving a higher dose of CD34 cells. These findings highlighted the fact that $\mathrm{T}$ cell depleted (TCD) HFD transplantation was a technically demanding procedure requiring experience and the results heavily depended on the CD34 cell content of the graft. The other major hindrance for its universal application was the high TRM associated with delayed IR. Whilst the major centres performing such procedures develop protocols and expertise in managing these complications, the ones performing TCD HFD transplants only occasionally were unlikely to achieve similar results.

\section{Natural Killer (NK) Cell Alloreactivity: A New Kid in the Block}

The focus of GVHD and GVL had remained on T cells until Ruggeri et al. highlighted the impact of natural killer (NK) cells in reduction of relapse in AML following CD34 selected PBSC grafts from haploidentical donors [25]. Since then, several groups have reported on the impact of NK cells in shaping the outcome of both haploidentical family donor and unrelated donor transplantation. The opinion has often been divided on this issue [26-28]. The last decade has witnessed an enormous effort in the understanding of NK cell biology within the context of allogeneic hematopoietic cell transplantation (HCT).

NK cells kill their target through direct cytotoxicity by engaging one or more activating receptors. However, the activating receptors are believed to be under the negative feedback control from inhibitory killer immunoglobulin-like 
receptors (KIRs). Cytotoxicity of NK cells in the steady state is under the constant negative feedback from inhibitory KIRs through binding to Self-Class $1 \mathrm{MHC}$ molecules. Several key KIR genes have been identified along with their putative ligands, whilst others remain unidentified. Biallelic polymorphism in HLA-C (positions 77 and 80 of heavy chain) denoted as $\mathrm{C}_{1}$ or $\mathrm{C}_{2}$ and restricted polymorphism in HLA-B (positions 77-83 in heavy chain) denoted as BW4 have been identified as ligands for KIR 2DL2/3, 2DL1, and 3DL1, respectively [29].

When NK cells from biallelic donor $\left(C_{1}\right.$ and $\left.C_{2}\right)$, for example, fail to find one of the alleles $\left(C_{1}\right.$ or $\left.C_{2}\right)$ in the recipient, a subset of donor NK cells tend to lose the inhibitory feedback and target the host hematopoietic cells vis-a-vis the leukemia cells in cytotoxic killing. This phenomenon (missing self-theory) was described by the Perugia group as the key event responsible for the cure of high risk leukemia following CD34 selected haploidentical graft [29, 30]. Several other models of NK alloreactivity have been postulated, yet none have been proven beyond surrogacy in the clinical setting [29-32].

In recent years, the focus has shifted to the repertoire of activating genes in the donor NK cells. Sivori et al. reported on the beneficial outcome of donor KIR2DS1 expression in conjunction with C2 allele in the recipient [33]. Furthermore Cooley et al. showed that KIR haplotypes and the specific genes related to $\mathrm{B}$ haplotype in the donor at centromeric or telomeric positions might have a favourable impact on the outcome of both unrelated and haploidentical HCT [34].

\section{Manipulating the Graft Further: Positive versus Negative Selection}

The seminal findings on NK alloreactivity along with development of immunomagnetic cell selection gave researchers in the field the options to rid the graft of CD3 and CD19 cells, leaving behind CD34, CD56, and other cell types [35]. The Tuebingen group reported on 46 children undergoing HFD HSCT with CD3+/CD19+ depleted graft in 2014 [36]. The engraftment was $88 \%$ with $20 \%$ TRM at 5 years. However, the incidences of both acute and chronic GVHD were higher with this approach, unlike that witnessed with CD34 selected grafts. The same group studied NK cell reconstitution in 59 patients undergoing CD3/19 depletion as compared to 42 patients undergoing CD34 selection [37]. They observed superior NK cell recovery and cytotoxicity with the former approach.

However, despite achieving a DFS of $45 \%$ to $80 \%$ when children were in CR, both TCD approaches were associated with dismal outcomes in more advanced diseases [18, 22, 36-38]. Employment of other TCD approaches in HFD transplantation for children with advanced leukemia did not result in improved outcome [39].

Further refinement of this approach took place with a new TCD method that removes $\alpha \beta+\mathrm{T}$ lymphocytes via a biotinylated anti-TCR $\alpha \beta$ antibody followed by an anti-biotin antibody conjugated to magnetic microbeads while retaining TCR $\gamma \delta+$ T lymphocytes, natural killer (NK) cells, and other cells in the graft [40]. This approach was based on the fact that the TCR $\alpha \beta$ T cells were primarily responsible for GVHD and that TCR $\gamma \delta$ T cells had potent antileukemia and antipathogen activity which, coupled with NK cells in the graft, would boost both antitumor and anti-infective potency of the graft. This approach has yielded excellent results in children with nonmalignant diseases and in those with acute leukemia in CR [41-44]. The IR was accelerated with this approach compared to the previous ones. The incidence of both acute and chronic GVHD remained low more akin to the CD3/CD19 depletion approach. However, the outcome of children not in CR remained dismal [43].

Another innovative approach from the Perugia group has taken graft manipulation a level further [45]. In accordance with the animal studies, they infused CD4+ CD25+ FoxP3+ regulatory $\mathrm{T}$ cell subpopulation (Tregs) on day -4 at $2 \times 10^{6} /$ $\mathrm{kg}$ following myeloablative conditioning [46]. This was followed by infusion of $>10 \times 10^{6} / \mathrm{kg}$ CD34 cells on day 0 along with $1 \times 10^{6} / \mathrm{kg}$ conventional T cells. This study was exclusively in adults and resulted in a DFS of $53 \%$ in patients with high risk leukemia, primarily in remission [46]. The authors claimed that this approach might reduce GVHD and yet augment the GVL effect. This approach is exciting but expensive and labor intensive.

Despite the encouraging results of TCD based approaches, two major caveats remain. Firstly, the approaches are technically demanding and expensive limiting its global application. Second, TCD based HSCT has uniformly yielded abysmal results in more advanced leukemia, particularly if not in remission [47].

\section{Unmanipulated Haploidentical HSCT: Changing the Paradigm in Adults, but What about Children?}

Two major approaches to HFD HSCT without graft manipulation in adults have changed the approach and outlook towards haploidentical transplantation in the last 5 years. The first approach pioneered by the Peking University group employed myeloablative conditioning with combined G-CSF stimulated marrow and PBSC grafts along with multiagent GVHD prophylaxis [48, 49]. Outcome data on 1210 transplants were reported in both adults and children with mostly ALL and AML with an impressive DFS of $67 \%$ and a NRM of $17 \%$ [50]. The incidences of acute and chronic GVHD were $40 \%$ and $50 \%$, respectively. The RI was only $17 \%$. The same group reported on the outcome of 212 children with a median age of 15 years with both AML and ALL [51]. They reported $100 \%$ engraftment with a NRM of $15 \%$ in those transplanted in CR1/CR2, but 25-40\% in those beyond CR2. The incidences of both acute and chronic GVHD were similar to those reported in the combined population, but grades 34 GVHD which occurred in 15\% of patients was identified as a risk factor for NRM. The RI was 7.2\% and 19\% in CR1 for AML and ALL, respectively, but was 2-4-fold higher beyond CR1. The overall DFS was 73\% for AML and $57 \%$ for ALL. In those beyond CR2, the DFS was $42 \%$ for AML and $22 \%$ for ALL. These results compare favourably with TCD 
approaches reported thus far. Not surprisingly, the incidences of both acute and chronic GVHD were much higher with this approach.

The other approach which was pioneered by the Johns Hopkins group involved use of posttransplantation cyclophosphamide (PTCY) [52]. This simple but unique concept is based on the fact that activated $\mathrm{T}$ cells are susceptible to high dose cyclophosphamide if administered in the window of 72 hours after graft infusion. The hematopoietic stem cells as well as quiescent $\mathrm{T}$ cells are spared of the cytotoxic effects of PTCY due to higher amount of aldehyde dehydrogenase $[53,54]$. It was shown in preclinical as well as the subsequent clinical studies that this approach resulted in $90 \%$ engraftment with very low incidences of both acute and chronic GVHD [55]. These studies were carried out in adults and the conditioning was nonmyeloablative (NMA) with marrow as the source of graft. The GVHD prophylaxis consisted of mycophenolate mofetil (MMF) for 35 days and tacrolimus for 180 days. In those grafted in CR1, the results were encouraging, but the ones with more advanced disease experienced very high incidences of relapse [56]. Subsequent studies on PTCY based HFD HSCT employing myeloablative conditioning reported better DFS with no significant increase in GVHD or NRM $[57,58]$. At the same time, several groups have used PBSC graft instead of BM and the outcomes have been similar in terms of engraftment and NRM with some increase in acute GVHD [59-61]. Thus, these studies have established PTCY based haploidentical HSCT as a frontrunner when it comes to alternate donor HSCT, to the extent that many argue in favour of PTCY based HFD HSCT ahead of MUD or UCBT [62-64].

Despite the impressive results in adults, the literature has been largely silent on the use of PTCY in children. One study from Japan employed a modified PTCY based approach on day +3 alone and GVHD prophylaxis with steroids and tacrolimus in 15 children, 9 of whom had advanced leukemia [65]. They reported a higher incidence of graft failure with lower conditioning intensity. Although $46 \%$ of the patients achieved a CR, the long term outcome remained dismal.

We had carried out a pilot study with PTCY based haploidentical PBSC transplantation on 20 children with advanced leukemia, 13 with refractory or relapsed AML and 7 with high risk ALL in CR1 [66]. A myeloablative conditioning with Fludarabine, Busulfan, and Melphalan was employed and GVHD prophylaxis consisted of MMF for 14-21 days and cyclosporine for 60 days with further 2 weeks of tapering. All engrafted promptly with 35\% experiencing grade 2-4 GVHD and 5\% having mild chronic GVHD. NRM was $20 \%$ at 1 year and this was associated with grade 3-4 GVHD, similar to that reported by the Chinese group [51]. However, it was of note that grade 3-4 GVHD occurred exclusively in those below the age of 10 years in our study. The above-mentioned study from Japan also documented GVHD in 6/8 evaluable patients below the age of 10 years [65]. In addition, we also experienced a higher incidence of early alloreactivity in the form of hemophagocytic syndrome (HPS) in children below 10 years of age [67].

\section{Why Are Younger Children at a Higher Risk of Early Alloreactivity following PTCY Based Haploidentical HSCT?}

This finding is indeed intriguing and counterintuitive on the face of it. The high incidence of early alloreactivity in the younger children undermines the basic principle of the PTCY approach and contradicts the prevailing concept that GVHD occurs with increasing age rather than the other way around. The relative contents of CD34+ cells and CD3+ T cells in our study were similar in both younger and older children and hence the higher T lymphocyte content of PBSC graft is unlikely to be solely responsible for the disparate outcome in the younger children [66]. Based on these findings, we hypothesised that the possible reason for early alloreactivity could be related to the failure of elimination of the alloreactive T cells by PTCY in younger children. To support this hypothesis, the pharmacokinetic (PK) studies on CY metabolism in children have been shown to be extremely variable [68]. In a study on 38 children between the ages of 2 and 15 years, there was significant interpatient variability as well as variable activation of CY to its active metabolites $[69,70]$. A pharmacokinetic study of high dose CY in children above the age of 10 years undergoing myeloablative conditioning for solid tumours did not reveal any impact of age on clearance or the volume of distribution of CY [71]. Extrapolating from the pharmacokinetic studies, this phenomenon might be explained by the reduced efficacy of PTCY in clearing alloreactive $\mathrm{T}$ cells in younger children, due to the variable metabolism of the drug in younger age group. Whether the alloreactivity would be less with marrow grafts is unclear due to the lack of data on the same. These findings once again serve as a reminder not to consider children as mere smaller adults and a regimen deemed successful in adults might not necessarily yield similar results in children.

\section{Choice of Graft for Children in CR1 Lacking a Matched Donor?}

In those in whom an allograft is recommended in CR1, traditionally, a MUD or UCBT from a cord unit with high cell dose and $\leq 2$ allele mismatches would generally be preferred for both AML and ALL. In conventional algorithm, a TCD graft from a HFD would be considered appropriate if none of the above is available. The relevant studies on HFD HSCT in children have been summarised in Tables 1 and 2. Whilst this approach is feasible and can produce impressive results in experienced hands, the procedure remains challenging to most of the world due to financial and technical demands associated with it. However, the cost of procuring a cord or a MUD graft is even more and the absence of GVHD and its prophylaxis or treatment following TCD HFD graft largely balances out the upfront financial burden in the long term along with an improved quality of life due to lack of immunosuppression and chronic GVHD. The best results with this approach are obtained in patients in complete remission, CR1 or CR2, rather than those not in CR or beyond CR2 $[18,20$, $36,39,43]$. The newer approaches to TCD such as TCR $\alpha \beta$ 


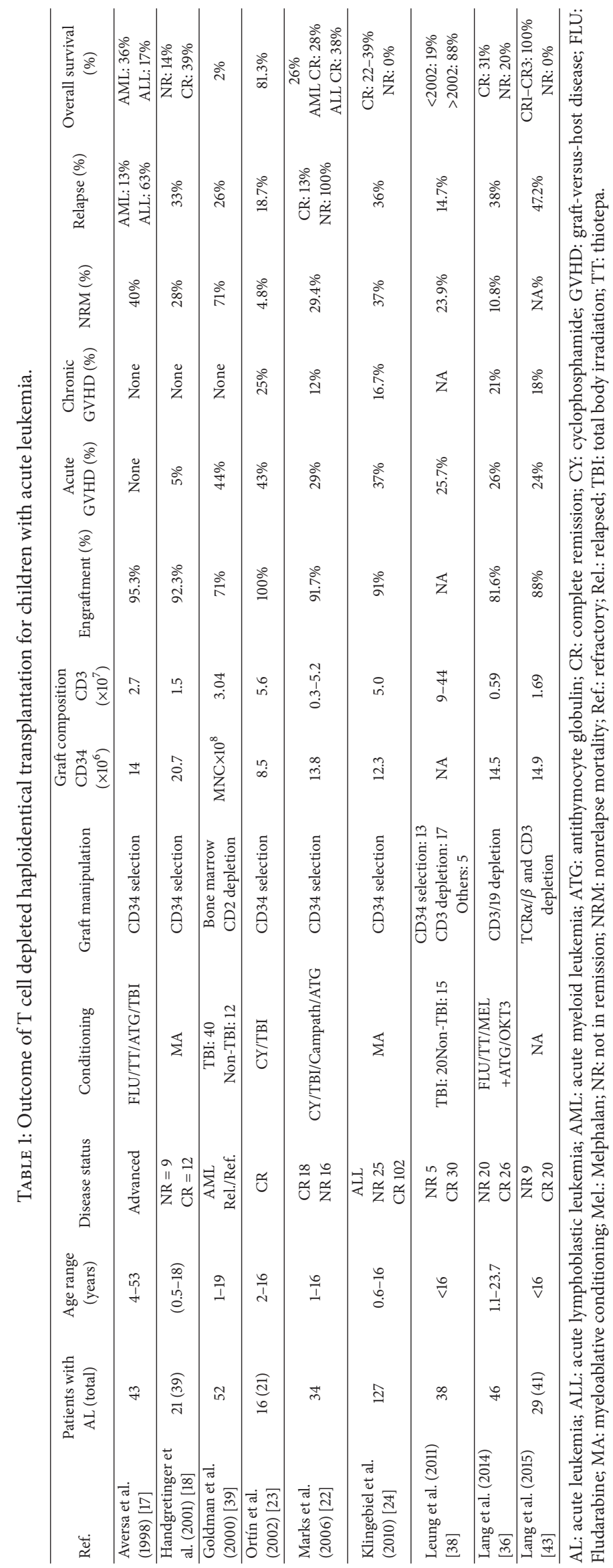




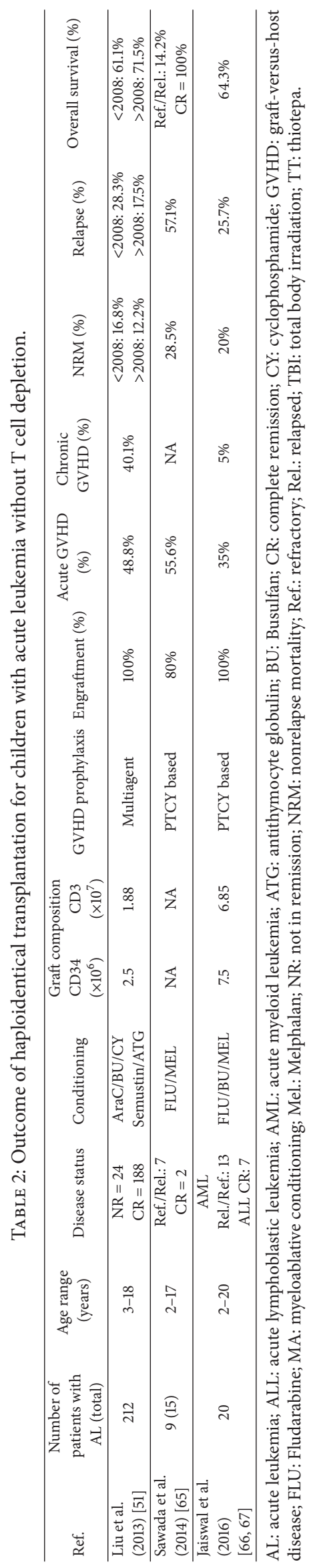


depletion might be more appropriate than CD34 selection due to the poor immune reconstitution associated with the latter resulting in significant infection associated mortality $[20,39,43,72]$. However, the data on the former is scanty and follow-ups are short to allow any definitive verdict in favour of either. Furthermore, NK cell alloreactivity plays an important role in reducing relapses for myeloid malignancies following TCD grafts in the HFD setting [32, 73]. The same is not established unequivocally in the context of ALL [73]. Some studies have suggested that NK cell alloreactivity might be effective in T cell ALL as well, whilst another study suggested an improved outcome in childhood ALL with a donor NK cell KIR B haplotype with higher B score [74].

The preferred modality of graft manipulation would be subject to the experience of individual centres with the main thrust on administering high number of CD34 cells, preferably in the range of $15-20 \times 10^{6} / \mathrm{kg}$. An NK alloreactive donor would be preferred as would be a maternal donor, if the graft is T cell depleted [75]. The issue of donor NK haplotype and $\mathrm{B}$ score might be relevant but remains uncertain pending further studies. However, if more than one NK alloreactive HFD is available, choosing one with a B haplotype and/or higher B score might be preferred. Although the data on NK cell alloreactivity is more robust in HFD transplants for AML, the limited data should not preclude the choice of the same in ALL.

If TCD is not feasible due to technical or financial reasons, should one opt for an unmanipulated graft and if so, what should dictate the choice of the donor? Given the limited data on non-TCD approaches, the recommendations would be more tailored to the individual situation. The study by the Chinese group has yielded impressive results in both AML and ALL in CR1. However, data is not available from other centres employing a similar approach and it remains unclear if the results would be similar in other ethnic groups. This is exemplified by a much higher incidence of HPS following both UCBT and HFD HSCT from Asia as compared to Europe and Northern America $[67,76]$. The data on PTCY based approach is limited, but early data indicates that this approach is best limited to children above the age of 10 years due to a higher risk of early alloreactivity [66].

The next issue that needs to be addressed is related to the choice of the haploidentical family donor. If the former approach is chosen, the choice of donor might be more definitive as donor issues have been extensively studied by the researchers from Peking University [50]. Interestingly, in direct contradiction to the data from TCD HFD [75], maternal donors were found to be associated with poorer outcomes. Three factors stood out in this analysis, donor gender, donor age, and noninherited maternal antigen (NIMA) mismatch. Thus, a NIMA mismatched younger male sibling would be a preferred donor followed by the father over mother or a sister. The same group had shown a detrimental effect of NK cell alloreactivity on the outcome, which was again contrary to the findings from TCD approach [77]. The same, however, cannot be extrapolated to other forms of unmanipulated HFD grafts and, pending further studies, a NIMA mismatched sibling donor might be a reasonable option. However, given the increased number of single child nuclear families, one might be left to choose between the parents. The Johns Hopkins group had shown that there might not be an impact of NK cell alloreactivity in the context of NMA PTCY based haploidentical transplantation [78]. Rather, a HFD with NK $\mathrm{B}$ haplotype might yield better results. This again remains unproven in the pediatric setting following myeloablative conditioning.

The current excellent results of TCD haploidentical HSCT could challenge the current hierarchical algorithm of alternate donor choice of MUD and UCBT in preference to HFD grafts, especially when the financial implications of the latter are more favourably balanced in the long term. It would not be unwise to assume that continued advances in the field of HFD HSCT might make this form of alternate donor HSCT the preferred option in the near future.

\section{The Choice of Graft beyond CR1/CR2}

The results of TCD approaches have been uniformly dismal even with newer methods of graft manipulation in these patients $[43,47]$ and a non-TCD approach might be preferred. Given the high risk of treatment failure, higher incidence of both acute and chronic GVHD following an unmanipulated graft might be more acceptable. Similar to TCD approaches, the choice would be centre specific with the main aim of the regimen directed at reducing both relapse and NRM. However, if a TCD approach is employed, this needs to be combined with immunotherapy. Whether infusion of NK cells or even $\gamma \delta$ T cells can improve the outcome remains to be seen but poses an exciting area of research [41, 79, 80]. The other approach being studied is the use of suicide gene modified T cells $[81,82]$. Thus, it might be prudent to enroll such patients in one of the trials employing any of these approaches. At our centre, we continue with Flu-BuMel conditioning and PBSC graft with PTCY and attenuated courses of both MMF and CSA in those above 10 years old. In those under the age of 10 years, we are currently enrolling patients with relapsed refractory leukemia in a study exploring inhibitors of T cell activation with PTCY to prevent early alloreactivity or TCD grafts with active immunotherapy.

\section{Optimizing NK Cell Mediated GVL Effect in Unmanipulated Haploidentical HSCT}

NK cell alloreactivity is unequivocally demonstrable following TCD haploidentical HSCT and yet has not been discernable with an unmanipulated graft. This paradox has never been addressed but undoubtedly deserves a closer look. There could be several explanations for this phenomenon. Ligand mismatches are not the only prerequisite for realising the antileukemia effect of alloreactive NK cells. Studies on HFD transplantation with unmanipulated graft have employed MMF as GVHD prophylaxis and have routinely employed GCSF after transplant. Both of these interventions compromise NK cell cytotoxicity $[83,84]$ and so does sirolimus [85]. On the other hand, CSA probably does not impair NK cell activity and might even augment it $[85,86]$. 
High dose CY has also been shown to enhance NK cell activation. Administration of high doses of CY improved the antitumor effect of IL-2 activated NK cells in animal models [87]. The cytotoxic activity of NK cells was increased by over $300 \%$ when they were incubated with CY. This effect was demonstrable after 2 hours and maximised after 8 hours of incubation [88]. In a clinical study on adoptive immunotherapy with haploidentical NK cells in patients with refractory AML, the use of high dose CY was associated with increased expansion of donor NK cells [89]. This was attributed to a marked rise in endogenous IL-15, a phenomenon not witnessed with low dose CY.

Thus, the combination of PTCY and CSA might provide the ideal platform to exploit the antileukemic potential of alloreactive NK cells, if the use of MMF or G-CSF could be limited. Furthermore, the use of PBSC graft rather than marrow might contribute to this phenomenon.

\section{Conclusion}

Haploidentical HSCT has come a long way since the initial failures in the 1980s [90]. The concepts of both the veto effect of CD34 cells when infused alone in large amounts and the utilisation of metabolic principles of cyclophosphamide in eradicating activated $\mathrm{T}$ cells immediately after transplantation have ushered a new era in alternate donor transplantation. Newer methods of graft manipulation with adoptive immunotherapy might pave the way for greater successes in the field of HFD transplantation for children with acute leukemia. At the same time, improving on the approaches to unmanipulated haploidentical HSCT is essential to realise the global potential of this procedure.

\section{Competing Interests}

The authors declare that there is no conflict of interests regarding the publication of this paper.

\section{References}

[1] A. Möricke, A. Reiter, M. Zimmermann et al., "Risk-adjusted therapy of acute lymphoblastic leukemia can decrease treatment burden and improve survival: treatment results of 2169 unselected pediatric and adolescent patients enrolled in the trial ALL-BFM 95," Blood, vol. 111, no. 9, pp. 4477-4489, 2008.

[2] H. Hasle, "A critical review of which children with acute myeloid leukaemia need stem cell procedures," British Journal of Haematology, vol. 166, no. 1, pp. 23-33, 2014.

[3] V. Rocha, J. Cornish, E. L. Sievers et al., "Comparison of outcomes of unrelated bone marrow and umbilical cord blood transplants in children with acute leukemia," Blood, vol. 97, no. 10, pp. 2962-2971, 2001.

[4] S. J. Lee, J. Klein, M. Haagenson et al., "High-resolution donorrecipient HLA matching contributes to the success of unrelated donor marrow transplantation," Blood, vol. 110, no. 13, pp. 45764583, 2007.

[5] L. Gragert, M. Eapen, E. Williams et al., "HLA match likelihoods for hematopoietic stem-cell grafts in the U.S. registry,"
The New England Journal of Medicine, vol. 371, no. 4, pp. 339348, 2014.

[6] M. Eapen, J. P. Klein, G. F. Sanz et al., "Effect of donor-recipient HLA matching at HLA A, B, C, and DRB1 on outcomes after umbilical-cord blood transplantation for leukaemia and myelodysplastic syndrome: a retrospective analysis," The Lancet Oncology, vol. 12, no. 13, pp. 1214-1221, 2011.

[7] M. Eapen, J. P. Klein, A. Ruggeri et al., "Impact of allele-level HLA matching on outcomes after myeloablative single unit umbilical cord blood transplantation for hematologic malignancy," Blood, vol. 123, no. 1, pp. 133-140, 2014.

[8] B. Oran and E. J. Shpall, "Allele-Level HLA cord blood matching matters," Blood, vol. 123, no. 1, pp. 8-9, 2014.

[9] B. Oran, K. Cao, R. M. Saliba et al., "Better allele-level matching improves transplant-related mortality after double cord blood transplantation," Haematologica, vol. 100, no. 10, pp. 1361-1370, 2015.

[10] B. E. Shaw, "The clinical implications of HLA mismatches in unrelated donor haematopoietic cell transplantation," International Journal of Immunogenetics, vol. 35, no. 4-5, pp. 367-374, 2008.

[11] M. R. Verneris, S. J. Lee, K. W. Ahn et al., "HLA mismatch is associated with worse outcomes after unrelated donor reducedintensity conditioning hematopoietic cell transplantation: an analysis from the center for international blood and marrow transplant research," Biology of Blood and Marrow Transplantation, vol. 21, no. 10, pp. 1783-1789, 2015.

[12] D. Weisdorf, S. Spellman, M. Haagenson et al., "Classification of HLA-matching for retrospective analysis of unrelated donor transplantation: revised definitions to predict survival," Biology of Blood and Marrow Transplantation, vol. 14, no. 7, pp. 748-758, 2008.

[13] R. L. Powles, H. E. M. Kay, H. M. Clink et al., "Mismatched family donors for bone-marrow transplantation as treatment for acute leukaemia," The Lancet, vol. 321, no. 8325, pp. 612-615, 1983.

[14] N. Or-Geva and Y. Reisner, "Megadose stem cell administration as a route to mixed chimerism," Current Opinion in Organ Transplantation, vol. 19, no. 4, pp. 334-341, 2014.

[15] N. Or-Geva and Y. Reisner, "Exercising 'veto' power to make haploidentical hematopoietic stem cell transplantation a safe modality for induction of immune tolerance," Regenerative Medicine, vol. 10, no. 3, pp. 239-242, 2015.

[16] Y. Reisner and M. F. Martelli, "Transplantation tolerance induced by 'mega dose' CD $34^{+}$cell transplants," Experimental Hematology, vol. 28, no. 2, pp. 119-127, 2000.

[17] F. Aversa, A. Tabilio, A. Velardi et al., "Treatment of high-risk acute leukemia with T-cell-depleted stem cells from related donors with one fully mismatched hla haplotype," The New England Journal of Medicine, vol. 339, no. 17, pp. 1186-1193, 1998.

[18] R. Handgretinger, T. Klingebiel, P. Lang et al., "Megadose transplantation of purified peripheral blood $\mathrm{CD} 34^{+}$progenitor cells from HLA-mismatched parental donors in children," Bone Marrow Transplantation, vol. 27, no. 8, pp. 777-783, 2001.

[19] F. Aversa, A. Terenzi, R. Felicini et al., "Haploidentical stem cell transplantation for acute leukemia," International Journal of Hematology, vol. 76, supplement 1, pp. 165-168, 2002.

[20] F. Aversa, Y. Reisner, and M. F. Martelli, “The haploidentical option for high-risk haematological malignancies," Blood Cells, Molecules, and Diseases, vol. 40, no. 1, pp. 8-12, 2008. 
[21] F. Aversa, "T cell depleted haploidentical transplantation: positive selection," Pediatric Reports, vol. 3, supplement 2, article el4, 2011.

[22] D. I. Marks, N. Khattry, M. Cummins et al., "Haploidentical stem cell transplantation for children with acute leukaemia," British Journal of Haematology, vol. 134, no. 2, pp. 196-201, 2006.

[23] M. Ortín, R. Raj, E. Kinning, M. Williams, and P. J. Darbyshire, "Partially matched related donor peripheral blood progenitor cell transplantation in paediatric patients adding fludarabine and antilymphocyte gamma-globulin," Bone Marrow Transplantation, vol. 30, no. 6, pp. 359-366, 2002.

[24] T. Klingebiel, J. Cornish, M. Labopin et al., "Results and factors influencing outcome after fully haploidentical hematopoietic stem cell transplantation in children with very high-risk acute lymphoblastic leukemia: Impact of center size: an analysis on behalf of the Acute Leukemia and Pediatric Disease Working Parties of the European Blood and Marrow Transplant group," Blood, vol. 115, no. 17, pp. 3437-3446, 2010.

[25] L. Ruggeri, M. Capanni, E. Urbani et al., "Effectiveness of donor natural killer cell alloreactivity in mismatched hematopoietic transplants," Science, vol. 295, no. 5562, pp. 2097-2100, 2002.

[26] E. J. Lowe, V. Turner, R. Handgretinger et al., "T-cell alloreactivity dominates natural killer cell alloreactivity in minimally T-cell-depleted HLA-non-identical paediatric bone marrow transplantation," British Journal of Haematology, vol. 123, no. 2, pp. 323-326, 2003.

[27] S. M. Davies, L. Ruggieri, T. DeFor et al., "Evaluation of KIR ligand incompatibility in mismatched unrelated donor hematopoietic transplants," Blood, vol. 100, no. 10, pp. 3825-3827, 2002.

[28] A. Bishara, D. De Santis, C. C. Witt et al., "The beneficial role of inhibitory KIR genes of HLA class I NK epitopes in haploidentically mismatched stem cell allografts may be masked by residual donor-alloreactive T cells causing GVHD," Tissue Antigens, vol. 63, no. 3, pp. 204-211, 2004.

[29] A. Moretta, C. Bottino, D. Pende et al., "Identification of four subsets of human CD3-CD16+ Natural Killer (NK) cells by the expression of clonally distributed functional surface molecules: correlation between subset assignment of NK clones and ability to mediate specific alloantigen recognition," Journal of Experimental Medicine, vol. 172, no. 6, pp. 1589-1598, 1990.

[30] L. Ruggeri, M. Capanni, M. Casucci et al., "Role of natural killer cell alloreactivity in HLA-mismatched hematopoietic stem cell transplantation," Blood, vol. 94, no. 1, pp. 333-339, 1999.

[31] K. C. Hsu, T. Gooley, M. Malkki et al., "KIR ligands and prediction of relapse after unrelated donor hematopoietic cell transplantation for hematologic malignancy," Biology of Blood and Marrow Transplantation, vol. 12, no. 8, pp. 828-836, 2006.

[32] L. Ruggeri, A. Mancusi, M. Capanni et al., "Donor natural killer cell allorecognition of missing self in haploidentical hematopoietic transplantation for acute myeloid leukemia: challenging its predictive value," Blood, vol. 110, no. 1, pp. 433-440, 2007.

[33] S. Sivori, S. Carlomagno, M. Falco, E. Romeo, L. Moretta, and A. Moretta, "Natural killer cells expressing the KIR2DS1activating receptor efficiently kill T-cell blasts and dendritic cells: implications in haploidentical HSCT," Blood, vol. 117, no. 16, pp. 4284-4292, 2011.

[34] S. Cooley, D. J. Weisdorf, L. A. Guethlein et al., "Donor selection for natural killer cell receptor genes leads to superior survival after unrelated transplantation for acute myelogenous leukemia," Blood, vol. 116, no. 14, pp. 2411-2419, 2010.
[35] R. C. Barfield, M. Otto, J. Houston et al., "A one-step largescale method for T- and B-cell depletion of mobilized PBSC for allogeneic transplantation," Cytotherapy, vol. 6, no. 1, pp. 1-6, 2004.

[36] P. Lang, H.-M. Teltschik, T. Feuchtinger et al., "Transplantation of CD3/CD19 depleted allografts from haploidentical family donors in paediatric leukaemia," British Journal of Haematology, vol. 165, no. 5, pp. 688-698, 2014.

[37] M. M. Pfeiffer, T. Feuchtinger, H.-M. Teltschik et al., "Reconstitution of natural killer cell receptors influences natural killer activity and relapse rate after haploidentical transplantation of T- and B-cell depleted grafts in children," Haematologica, vol. 95, no. 8, pp. 1381-1388, 2010.

[38] W. Leung, D. Campana, J. Yang et al., "High success rate of hematopoietic cell transplantation regardless of donor source in children with very high-risk leukemia," Blood, vol. 118, no. 2, pp. 223-230, 2011.

[39] F. D. Goldman, S. L. Rumelhart, P. DeAlacron et al., "Poor outcome in children with refractory/relapsed leukemia undergoing bone marrow transplantation with mismatched family member donors," Bone Marrow Transplantation, vol. 25, no. 9, pp. $943-$ $948,2000$.

[40] M. Schumm, P. Lang, W. Bethge et al., "Depletion of T-cell receptor $\alpha / \beta$ and CD19 positive cells from apheresis products with the CliniMACS device," Cytotherapy, vol. 15, no. 10, pp. 1253-1258, 2013.

[41] I. Airoldi, A. Bertaina, I. Prigione et al., " $\gamma \delta$ T-cell reconstitution after HLA-haploidentical hematopoietic transplantation depleted of TCR- $\alpha \beta^{+} / \mathrm{CD} 19^{+}$lymphocytes," Blood, vol. 125, no. 15, pp. 2349-2358, 2015.

[42] A. Bertaina, P. Merli, S. Rutella et al., "HLA-haploidentical stem cell transplantation after removal of $\alpha \beta+\mathrm{T}$ and $\mathrm{B}$ cells in children with nonmalignant disorders," Blood, vol. 124, no. 5, pp. 822-826, 2014.

[43] P. Lang, T. Feuchtinger, H. M. Teltschik et al., "Improved immune recovery after transplantation of TCR $\alpha \beta / \mathrm{CD} 19$-depleted allografts from haploidentical donors in pediatric patients," Bone Marrow Transplantation, vol. 50, supplement 2, pp. S6S10, 2015.

[44] F. Locatelli, A. Bauquet, G. Palumbo, F. Moretta, and A. Bertaina, "Negative depletion of $\alpha / \beta^{+}$T cells and of CD19+ B lymphocytes: a novel frontier to optimize the effect of innate immunity in HLA-mismatched hematopoietic stem cell transplantation," Immunology Letters, vol. 155, no. 1-2, pp. 21-23, 2013.

[45] M. F. Martelli, M. D. Ianni, L. Ruggeri et al., "Next generation HLA-haploidentical HSCT," Bone Marrow Transplantation, vol. 5, supplement 2, pp. S63-S66, 2015.

[46] M. F. Martelli, M. D. Ianni, L. Ruggeri et al., "HLA-haploidentical transplantation with regulatory and conventional Tcell adoptive immunotherapy prevents acute leukemia relapse," Blood, vol. 124, no. 4, pp. 638-644, 2014.

[47] F. Aversa, "T-cell depletion: from positive selection to negative depletion in adult patients," Bone Marrow Transplantation, vol. 50, pp. S11-S13, 2015.

[48] Y.-Q. Sun, J. Wang, Q. Jiang et al., "Haploidentical hematopoietic SCT may be superior to conventional consolidation/maintenance chemotherapy as post-remission therapy for high-risk adult ALL," Bone Marrow Transplantation, vol. 50, no. 1, pp. 2025, 2015.

[49] C.-H. Yan, Q. Jiang, J. Wang et al., "Superior survival of unmanipulated haploidentical hematopoietic stem cell transplantation compared with chemotherapy alone used as post-remission 
therapy in adults with standard-risk acute lymphoblastic leukemia in first complete remission," Biology of Blood and Marrow Transplantation, vol. 20, no. 9, pp. 1314-1321, 2014.

[50] Y. Wang, Y.-J. Chang, L.-P. Xu et al., "Who is the best donor for a related HLA haplotype-mismatched transplant?" Blood, vol. 124, no. 6, pp. 843-850, 2014.

[51] D.-H. Liu, L.-P. Xu, K.-Y. Liu et al., "Long-term outcomes of unmanipulated haploidentical HSCT for paediatric patients with acute leukaemia," Bone Marrow Transplantation, vol. 48, no. 12, pp. 1519-1524, 2013.

[52] P. V. O’Donnell, L. Luznik, R. J. Jones et al., "Nonmyeloablative bone marrow transplantation from partially HLA-mismatched related donors using posttransplantation cyclophosphamide," Biology of Blood and Marrow Transplantation, vol. 8, no. 7, pp. 377-386, 2002.

[53] C. G. Kanakry, S. Ganguly, M. Zahurak et al., "Aldehyde dehydrogenase expression drives human regulatory $\mathrm{T}$ cell resistance to posttransplantation cyclophosphamide," Science Translational Medicine, vol. 5, no. 211, Article ID 211ra157, 2013.

[54] L. Luznik, S. Jalla, L. W. Engstrom, R. Lannone, and E. J. Fuchs, "Durable engraftment of major histocompatibility complexincompatible cells after nonmyeloablative conditioning with fludarabine, low-dose total body irradiation, and posttransplantation cyclophosphamide," Blood, vol. 98, no. 12, pp. 3456-3464, 2001.

[55] L. Luznik, P. V. O’Donnell, H. J. Symons et al., "HLA-haploidentical bone marrow transplantation for hematologic malignancies using nonmyeloablative conditioning and high-dose, posttransplantation cyclophosphamide," Biology of Blood and Marrow Transplantation, vol. 14, no. 6, pp. 641-650, 2008.

[56] S. R. McCurdy, J. A. Kanakry, M. M. Showel et al., "Risk-stratified outcomes of nonmyeloablative HLA-haploidentical BMT with high-dose posttransplantation cyclophosphamide," Blood, vol. 125, no. 19, pp. 3024-3031, 2015.

[57] A. M. Raiola, A. Dominietto, A. Ghiso et al., "Unmanipulated haploidentical bone marrow transplantation and posttransplantation cyclophosphamide for hematologic malignancies after myeloablative conditioning," Biology of Blood and Marrow Transplantation, vol. 19, no. 1, pp. 117-122, 2013.

[58] S. R. Solomon, C. A. Sizemore, M. Sanacore et al., “TBI-based myeloablative haploidentical stem cell transplantation is a safe and effective alternative to unrelated donor transplantation in patients without matched sibling donors," Biology of Blood and Marrow Transplantation, vol. 124, no. 21, p. 426, 2015.

[59] L. Castagna, R. Crocchiolo, S. Furst et al., "Bone marrow compared with peripheral blood stem cells for haploidentical transplantation with a nonmyeloablative conditioning regimen and post-transplantation cyclophosphamide," Biology of Blood and Marrow Transplantation, vol. 20, no. 5, pp. 724-729, 2014.

[60] K. Raj, A. Pagliuca, K. Bradstock et al., "Peripheral blood hematopoietic stem cells for transplantation of hematological diseases from related, haploidentical donors after reduced-intensity conditioning," Biology of Blood and Marrow Transplantation, vol. 20, no. 6, pp. 890-895, 2014.

[61] S. R. Solomon, C. A. Sizemore, M. Sanacore et al., "Haploidentical transplantation using $\mathrm{T}$ cell replete peripheral blood stem cells and myeloablative conditioning in patients with high-risk hematologic malignancies who lack conventional donors is well tolerated and produces excellent relapse-free survival: results of a prospective phase II trial," Biology of Blood and Marrow Transplantation, vol. 18, no. 12, pp. 1859-1866, 2012.
[62] A. Bashey, X. Zhang, C. A. Sizemore et al., "T-cell-replete HLAhaploidentical hematopoietic transplantation for hematologic malignancies using post-transplantation cyclophosphamide results in outcomes equivalent to those of contemporaneous HLA-matched related and unrelated donor transplantation," Journal of Clinical Oncology, vol. 31, no. 10, pp. 1310-1316, 2013.

[63] S. O. Ciurea, M.-J. Zhang, A. A. Bacigalupo et al., "Haploidentical transplant with posttransplant cyclophosphamide vs matched unrelated donor transplant for acute myeloid leukemia," Blood, vol. 126, no. 8, pp. 1033-1040, 2015.

[64] A. M. Raiola, A. Dominietto, C. di Grazia et al., "Unmanipulated haploidentical transplants compared with other alternative donors and matched sibling grafts," Biology of Blood and Marrow Transplantation, vol. 20, no. 10, pp. 1573-1579, 2014.

[65] A. Sawada, M. Shimizu, K. Isaka et al., "Feasibility of HLAhaploidentical hematopoietic stem cell transplantation with post-transplantation cyclophosphamide for advanced pediatric malignancies," Pediatric Hematology and Oncology, vol. 31, no. 8, pp. 754-764, 2014.

[66] S. R. Jaiswal, A. Chakrabarti, S. Chatterjee et al., "Haploidentical peripheral blood stem cell transplantation with posttransplantation cyclophosphamide in children with advanced acute leukemia with fludarabine-, busulfan-, and melphalanbased conditioning," Biology of Blood and Marrow Transplantation, vol. 22, no. 3, pp. 499-504, 2016.

[67] S. R. Jaiswal, A. Chakrabarti, S. Chatterjee, S. Bhargava, K. Ray, and S. Chakrabarti, "Hemophagocytic syndrome following haploidentical peripheral blood stem cell transplantation with post-transplant cyclophosphamide," International Journal of Hematology, vol. 103, no. 2, pp. 234-242, 2016.

[68] S. M. Yule, A. V. Boddy, M. Cole et al., "Cyclophosphamide metabolism in children," Cancer Research, vol. 55, no. 4, pp. 803-809, 1995.

[69] S. M. Yule, A. V. Boddy, M. Cole et al., "Cyclophosphamide pharmacokinetics in children," British Journal of Clinical Pharmacology, vol. 41, no. 1, pp. 13-19, 1996.

[70] S. M. Yule, L. Price, M. Cole, A. D. J. Pearson, and A. V. Boddy, "Cyclophosphamide metabolism in children following a 1-h and a 24-h infusion," Cancer Chemotherapy and Pharmacology, vol. 47, no. 3, pp. 222-228, 2001.

[71] G. Chinnaswamy, J. Errington, A. Foot, A. V. Boddy, G. J. Veal, and M. Cole, "Pharmacokinetics of cyclophosphamide and its metabolites in paediatric patients receiving high-dose myeloablative therapy," European Journal of Cancer, vol. 47, no. 10, pp. 1556-1563, 2011.

[72] R. Handgretinger, X. Chen, M. Pfeiffer et al., "Cellular immune reconstitution after haploidentical transplantation in children," Biology of Blood and Marrow Transplantation, vol. 14, no. 1, pp. 59-65, 2008.

[73] F. Locatelli, D. Pende, M. C. Mingari et al., "Cellular and molecular basis of haploidentical hematopoietic stem cell transplantation in the successful treatment of high-risk leukemias: role of alloreactive NK cells," Frontiers in Immunology, vol. 4, article 15, 2013.

[74] L. Oevermann, S. U. Michaelis, M. Mezger et al., "KIR B haplotype donors confer a reduced risk for relapse after haploidentical transplantation in children with ALL," Blood, vol. 124, no. 17, pp. 2744-2747, 2014.

[75] M. Stern, L. Ruggeri, A. Mancusi et al., "Survival after T celldepleted haploidentical stem cell transplantation is improved using the mother as donor," Blood, vol. 112, no. 7, pp. 2990-2995, 2008. 
[76] S. Takagi, K. Masuoka, N. Uchida et al., "High incidence of haemophagocytic syndrome following umbilical cord blood transplantation for adults," British Journal of Haematology, vol. 147, no. 4, pp. 543-553, 2009.

[77] X.-J. Huang, X.-Y. Zhao, D.-H. Liu, K.-Y. Liu, and L.-P. Xu, "Deleterious effects of KIR ligand incompatibility on clinical outcomes in haploidentical hematopoietic stem cell transplantation without in vitro T-cell depletion," Leukemia, vol. 21, no. 4, pp. 848-851, 2007.

[78] Y. L. Kasamon, L. Luznik, M. S. Leffell et al., "Nonmyeloablative HLA-haploidentical bone marrow transplantation with highdose posttransplantation cyclophosphamide: effect of HLA disparity on outcome," Biology of Blood and Marrow Transplantation, vol. 16, no. 4, pp. 482-489, 2010.

[79] F. Locatelli, P. Merli, and S. Rutella, "At the bedside: innate immunity as an immunotherapy tool for hematological malignancies," Journal of Leukocyte Biology, vol. 94, no. 6, pp. 11411157, 2013.

[80] H. Norell, A. Moretta, B. Silva-Santos, and L. Moretta, "At the bench: preclinical rationale for exploiting NK cells and $\gamma \delta \mathrm{T}$ lymphocytes for the treatment of high-risk leukemias," Journal of Leukocyte Biology, vol. 94, no. 6, pp. 1123-1139, 2013.

[81] R. Greco, G. Oliveira, M. T. Stanghellini et al., "Improving the safety of cell therapy with the TK-suicide gene," Frontiers in Pharmacology, vol. 6, article 95, 2015.

[82] G. Oliveira, E. Ruggiero, M. T. L. Stanghellini et al., "Tracking genetically engineered lymphocytes long-term reveals the dynamics of T cell immunological memory," Science Translational Medicine, vol. 7, no. 317, Article ID 317ra198, 2015.

[83] K. Ohata, J. L. Espinoza, X. Lu, Y. Kondo, and S. Nakao, "Mycophenolic acid inhibits natural killer cell proliferation and cytotoxic function: a possible disadvantage of including mycophenolate mofetil in the graft-versus-host disease prophylaxis regimen," Biology of Blood and Marrow Transplantation, vol. 17, no. 2, pp. 205-213, 2011.

[84] L. Schlahsa, Y. Jaimes, R. Blasczyk, and C. Figueiredo, "Granulocyte-colony-stimulatory factor: a strong inhibitor of natural killer cell function," Transfusion, vol. 51, no. 2, pp. 293-305, 2011.

[85] L.-E. Wai, M. Fujiki, S. Takeda, O. M. Martinez, and S. M. Krams, "Rapamycin, but not cyclosporine or FK506, alters natural killer cell function," Transplantation, vol. 85, no. 1, pp. 145149, 2008.

[86] M. T. Kasaian and C. A. Biron, "Cyclosporin A inhibition of interleukin 2 gene expression, but not natural killer cell proliferation, after interferon induction in vivo," Journal of Experimental Medicine, vol. 171, no. 3, pp. 745-762, 1990.

[87] R. H. Goldfarb, M. Ohashi, K. W. Brunson et al., "Augmentation of IL-2 activated natural killer cell adoptive immunotherapy with cyclophosphamide," Anticancer Research, vol. 18, no. 3, pp. 1441-1446, 1998.

[88] B. Sharma and N. D. Vaziri, "Augmentation of human natural killer cell activity by cyclophosphamide in vitro," Cancer Research, vol. 44, no. 8, pp. 3258-3261, 1984.

[89] J. S. Miller, Y. Soignier, A. Panoskaltsis-Mortari et al., "Successful adoptive transfer and in vivo expansion of human haploidentical NK cells in patients with cancer," Blood, vol. 105, no. 8, pp. 3051-3057, 2005.

[90] Y. Reisner, F. Aversa, and M. F. Martelli, "Haploidentical hematopoietic stem cell transplantation: state of art," Bone Marrow Transplantation, vol. 50, supplement 2, pp. S1-S5, 2015. 


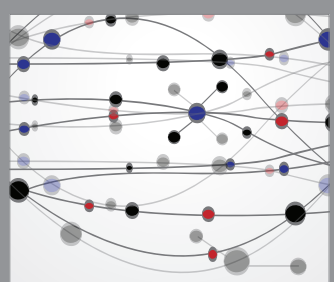

The Scientific World Journal
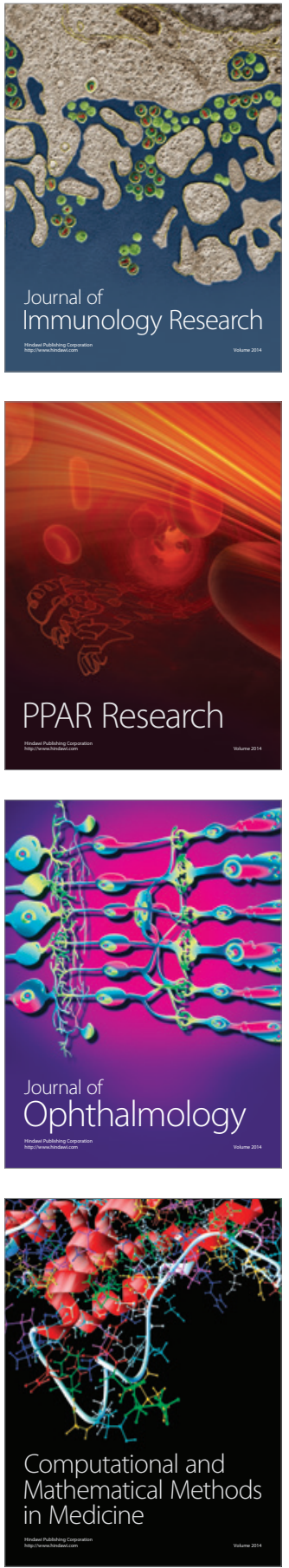

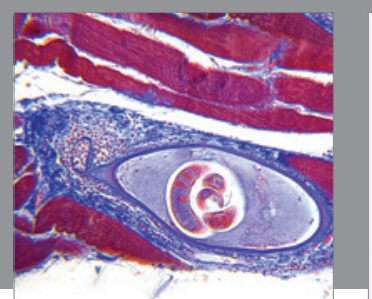

Gastroenterology Research and Practice

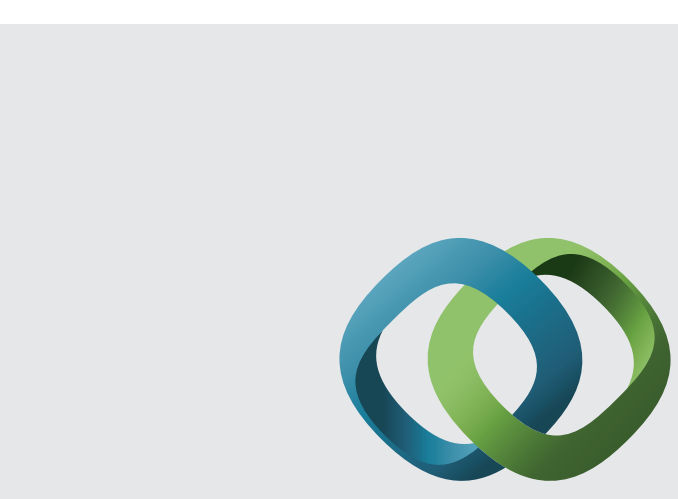

\section{Hindawi}

Submit your manuscripts at

http://www.hindawi.com
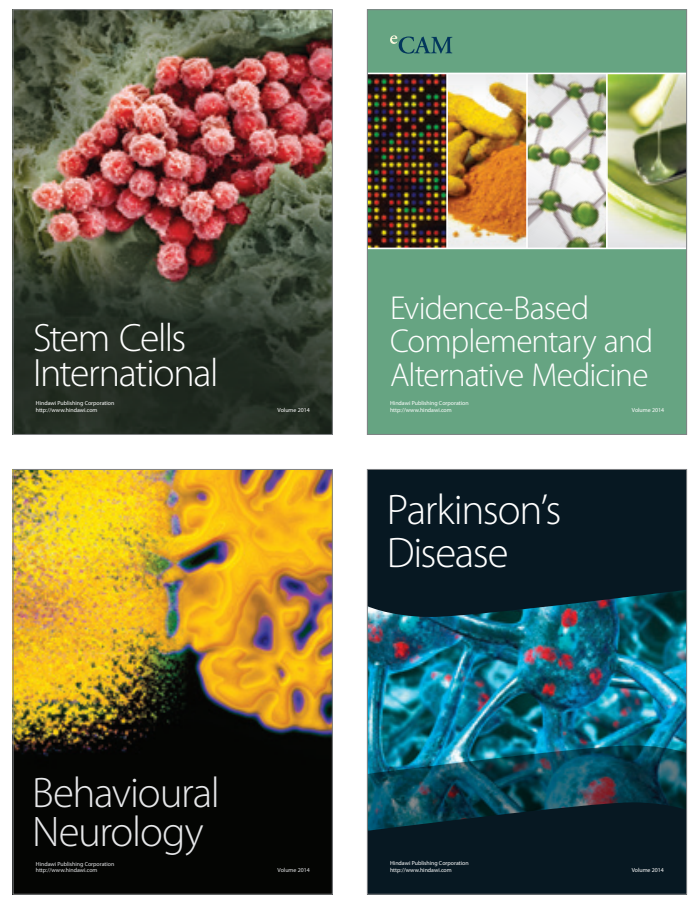
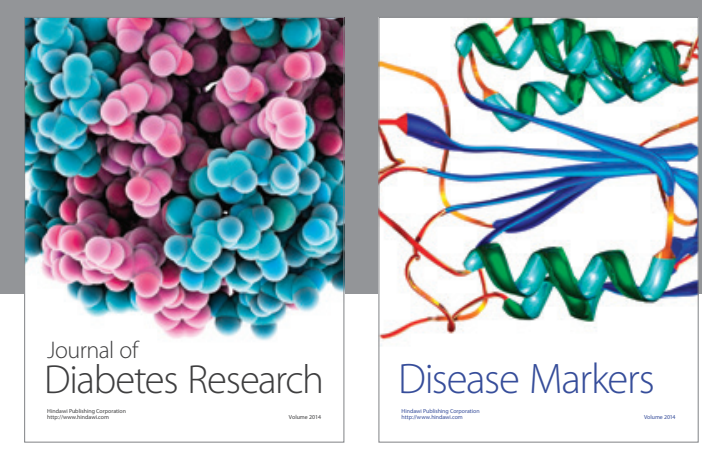

Disease Markers
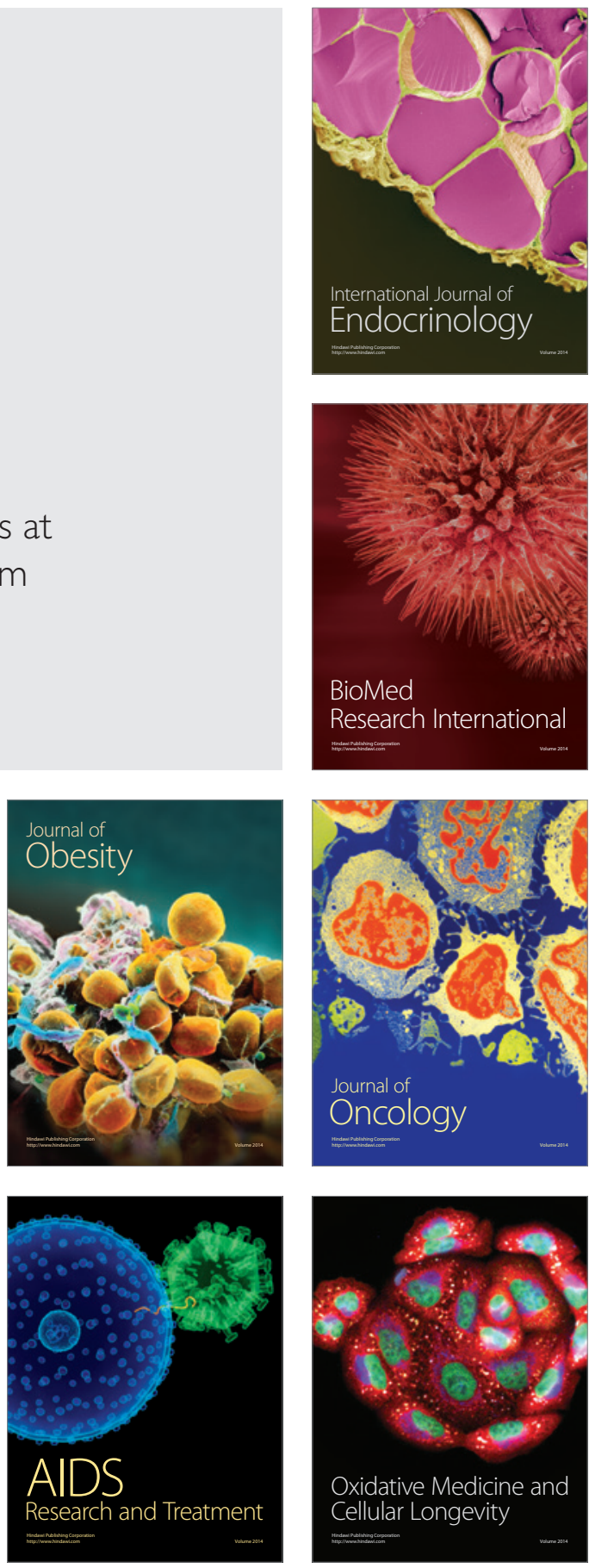